\title{
Evaluation of over the counter vaginal lubricants Nonoxynol-9 and KY Jelly as potential inducer of proinflammatory cytokines in human immune cells
}

\author{
Aladin Siddig a, ${ }^{*}$, Richard Addo b, and Kwame Yeboah c \\ a University of Charleston School of Pharmacy, Charleston, WV 25304, USA \\ b Union University School of Pharmacy, Jackson, TN 38305, USA \\ b Harding University College of Pharmacy, Arkansas 72149, USA \\ *Corresponding author at: University of Charleston School of Pharmacy, Charleston, WV 25304, USA. \\ Tel.: +1.304.3574352; fax: +1.304.3574868. E-mail address: aladinsiddag@ucwv.edu (A. Siddig).
}

\section{ARTICLE INFORMATION}

Received: 04 March 2013

Received in revised form: 21 March 2013

Accepted: 30 March 2013

Online: 30 June 2013

\section{KEYWORDS}

\section{KY Jelly}

Nonoxymol-9

Vaginal Lubricants

Anti-HIV-1 products

Topical Microbicides

Proinflammatory cytokines

\section{ABSTRACT}

Topical microbicides such as Nonoxynol-9 (N9) and KY jelly have been used as vaginal microbicides in clinical trials as anti-HIV-1 products. The objective of this study was to determine if the use of these microbicides may cause production of proinflammatory cytokines in vitro. Such cytokines include, tumor necrosis factor- $\alpha$ (TNF- $\alpha$ ), interlukin-1 $\beta$ (IL$1 \beta$ ), interlukin-6 (IL-6), and Interlukin-8 (IL-8). Cellular viability was determined for the peripheral blood mononuclear cells (PBMCs) and Macrophages by culturing them for 24 hours in the presence of serial dilutions of product or placebo. Product and placebo dilutions that gave culture viabilities of $\geq 60 \%$ compared to control cultures were considered to be nontoxic. The non-toxic dilutions were 1:100 for KY jelly in both PBMCs and Macrophages. Whereas, the non-toxic dilutions were 1:1000 for N9 in both PBMCs and Macrophages. The values of IL1 $\beta$, TNF- $\alpha$, were $0.1<2.0 \mathrm{pg} / \mathrm{mL}$ for both PBMCs and macrophages. The levels of IL-8 ranged between $9-13 \mathrm{pg} / \mathrm{mL}$ and $12-16 \mathrm{pg} / \mathrm{mL}$ for PBMCs and macrophages, respectively. The levels of IL- 6 were $0.11 \mathrm{~g} / \mathrm{mL}$ and $0.019 \mathrm{pg} / \mathrm{mL}$ for PBMCs and macrophages, respectively. The results were not statistically significant in difference for IL- $1 \beta$, IL-6, and IL-8 ( $p>0.001$ ) when N9 was compared to KY jelly. However, the results were significant when comparing N9 to KY jelly for TNF- $\alpha(\mathrm{p}<0.001)$ production. The two microbicides tested in their non-toxic formulation in the two models of PBMCs and macrophages showed relatively low levels of IL$1 \mathrm{~b}$, TNF- $\alpha$ and IL- 6 . This indicated safety and low toxicity of these microbicide in terms of cytokines release. On the other hand, IL-8 has shown relatively higher levels in all microbicide tested in their non-toxic formulation.

\section{Introduction}

The HIV epidemic continues throughout the world and is accelerating in many areas despite almost 30 years of research and prevention efforts. Approximately 34 million people worldwide are carriers of HIV in 2009 with $95 \%$ of these people living in developing countries; in particular, many African countries have HIV prevalence as high as $36 \%$. The majority of all adult HIV-1 transmissions occur through heterosexual intercourse [1]. This indicates that additional prevention methods are needed in combination with the riskreduction counseling programs and condom promotion methods, which already exist. Therefore, the identification of an affordable vaginal microbicide that is safe and decreases the risk of HIV transmission is extremely important and should be considered a high priority.

Currently, there is no commercially available vaginal microbicide with proven anti-HIV efficacy. However, it is a pivotal time in HIV microbicide research, in which more resources are becoming available and progress is being made in the field. For example, the well-known spermicide Nonoxynol-9 (N9) had been shown to inactivate HIV [2] and thus became popular for use in safe-sex practices. Recent studies, however, have shown that N-9 actually increases the risk of HIV-1 transmission, possibly due to increased HIV shedding and increased recruitment of CD4+ target cells associated with N-9 induced vaginal inflammation [3]. This finding may be responsible for the combination of N-9 with KY jelly as a modified vaginal lubricant. Although microbicides are not yet being distributed and sold, several microbicide candidates are actively being tested for anti-HIV activity in phase I and phase II clinical trials [4].

Over the counter (OTC) vaginal lubricants that fall under the safest category of the Food and Drug Administration (FDA) are also worthy for study because they do not cause irritation upon repeated use, readily available, inexpensive, widely accepted by the public, and would provide women with choice if their male partners do not choose to wear condoms [5]. Examples of OTC lubricants include Astroglide and KY Jelly.

The ideal microbicide or lubricant should block HIV infection without inducing mucosal irritation in the genital tract. It should also have low toxicity for cells and the natural microbial flora in the female genital tract. Additionally, it must be affordable and readily available to be distributed. Therefore any topical microbicide approved for human use will need to be first evaluated for epithelial toxicity and primary immune cells, because the product will come in direct contact with vaginal or rectal mucosal surfaces.

Important to the study of microbicides is an understanding of the biology of sexual transmission. It was initially reported that cell-free HIV in seminal fluid was the major source of maleto-female transmission, but recent studies suggest that virus- 
infected leukocytes (CD4+ mononuclear cells) in semen may also cause infections [6-12]. In addition, it has been found that most of the infectious virus in body fluids and secretions are cell-associated [13-16]. These infected leukocytes have the potential of directly infecting mucosal epithelial cells or penetrating the epithelial layer [6-11]. In contrast, genital mucosa exposed to cell-free virus has very low infectivity for epithelial cells lacking the CD4 receptor. Therefore, leukocytes may play a role in sexual transmission of HIV and they must be considered in the development of an effective microbicide [17].

The objectives of this study were:

- To determine whether OTC lubricants and candidate microbicides (N9-KY) are cytotoxic to a major target for HIV infection, peripheral blood mononuclear cells (PBMCs), macrophages, and epithelial Caco2 cells. Cytotoxicity was assessed using cell TiterGlo assay measuring the levels of cellular ATP production.

- To determine if topical microbicides could induce genital tract inflammation and thus increase the likelihood of HIV transmission by measuring the levels of tumor necrosis factor (TNF- $\alpha$ ), interleukin-1 $\beta$, interleukin-6, and interleukin-8 in PBMCs and macrophage cell cultures after exposure to topical microbicides.

\section{Experimental}

Epithelial cell lines were obtained from the American Type Culture Collection (Manassas, VA). Urogenital epithelial cell lines, ME-180 [18] and HEC-1-A [19], were grown in McCoy's $5 \mathrm{~A}$ supplemented with $10 \%$ fetal bovine serum, $100 \mathrm{~g} / \mathrm{mL}$ streptomycin, $100 \mathrm{U} / \mathrm{ml}$ penicillin, and $100 \mathrm{mM}$ L-glutamine. Colorectal epithelial cell lines, Caco-2 [20], were grown in DMEM (C-DMEM) supplemented with $10 \%$ fetal bovine serum, $0.1 \mathrm{mM}$ nonessential amino acids, $1 \mathrm{mM}$ sodium pyruvate, 100 $\mathrm{g} / \mathrm{mL}$ streptomycin, $100 \mathrm{U} / \mathrm{mL}$ penicillin, and $100 \mathrm{mM} \mathrm{L}-$ glutamine. The epithelial cell lines were maintained at $37{ }^{\circ} \mathrm{C}$, $7 \% \mathrm{CO}_{2}$.

Normal human PBMCs were obtained by leukophoresis from HIV-1-negative blood donors, purified by differential centrifugation, and stored in the gas phase of liquid nitrogen until needed. PBMCs were CD8-depleted using anti-CD8conjugated magnetic beads (Dynal, Lake Success, NY) according to the manufacturer's instructions. The CD8-depleted PBMCs were stimulated for 3 days at $37{ }^{\circ} \mathrm{C}, 7 \% \mathrm{CO}_{2}$ in C-RPMI supplemented with $10 \%$ IL-2 and 0.5 or $1.0 \mu \mathrm{g} / \mathrm{mL}$ phytohemagglutinin-P. After 3 days, the PBMCs were washed and suspended in C-RPMI with $10 \%$ IL-2. Human monocytes (Advanced Biotechnologies, Inc., Columbia, MD) were allowed to differentiate to Macrophages and were maintained in DMEM supplemented with $20 \%$ heat-inactivated fetal calf serum, $10 \%$ human $\mathrm{AB}$ serum, $100 \mu \mathrm{g} / \mathrm{mL}$ streptomycin, $100 \mathrm{U} / \mathrm{mL}$ penicillin, and $100 \mathrm{mM}$ L-glutamine at $37{ }^{\circ} \mathrm{C}, \quad 7 \% \mathrm{CO}_{2}$. Macrophages were maintained in the same media and culture conditions.

$\mathrm{K}-\mathrm{Y} \AA$ plus nonoxynol-9 (KY-N9; Ortho-McNeil Pharmaceuticals, Inc., Raritan, NJ) and K-Y® Jelly (McNeil-PPC, Inc., Skillman, NJ) are approved over-the-counter (OTC) products.

\subsection{Methods}

\subsubsection{Epithelial Caco2 cells}

Fifty thousand cells were added per well in a 96-well plate. After an overnight incubation to allow the cells to adhere to the well, dilutions of the products or their placebos were added to the wells in triplicate for either 24 or 2 hours per day for 5 days. For the 5 day exposure, the products or placebos were diluted in the appropriate epithelial cell medium and added to the culture for 2 hours and then washed twice with phosphatebuffered saline. Fresh medium was reapplied. Wells containing cells alone were assayed in triplicate and served as controls. To determine culture viability, cellular ATP concentrations were measured using the CellTiter-Glo ${ }^{\mathrm{TM}}$ assay (Promega Corp. Madison, WI) according to the manufacturer's instructions. Culture ATP-dependent luminescence was measured on a DYNEX MLX instrument (Franklin, MA) and reported as relative light units (RLU). The RLU ratio of test cultures and control cultures was used to assess cellular viability.

\subsubsection{Primary Immune Cells "PBMCs and Macrophages"}

Activated PBMCs were mixed with serial dilutions of product or placebo in medium or medium alone (control), and $1 \times 10^{6}$ cells were placed in a 24 well plate. Test and control cultures were set-up in triplicate. After 24 hours, cultures were gently mixed and $100 \mu \mathrm{L}$ was removed for testing. Cell viability was determined as described above. Macrophages were incubated with serial dilutions of a product or placebo in medium or medium alone (control) and cultured in a 24 well plate. Test and control cultures were set-up in duplicate. After 24 hours, media or product dilutions were removed and the wells were washed twice with fresh medium before $500 \mu \mathrm{L}$ of reconstituted CellTiter-Glo ${ }^{\mathrm{TM}}$ reagent was added to each well. The plate was placed on a horizontal rocker for 30 minutes. Viability was determined as described above.

\subsubsection{In-vitro Inflammation induction studies}

PBMCs or Macrophages $\left(1 \times 10^{6} /\right.$ well $)$ were incubated in medium containing $(1.0 \mathrm{~mL}$ media/well) and a nontoxic concentration of product or placebo or media alone (control) for 4 hours. The PBMCs were then cultured in medium alone at $1 \times 10^{6}$ cells $/ \mathrm{mL} /$ well in a 24 well plate for 4 hours. Each product, placebo, and control was tested in triplicate. The product and placebo were exposed to cells in their non-toxic as well as toxic forms (Table 1). Culture supernatants were stored at $-80{ }^{\circ} \mathrm{C}$ until they were assayed for proinflammatory cytokines using Human QuantikinesR HS cytokines immunoassay kit (R \& D systems, Minneapolis, MN). The epithelial cell lines and the primary immune cells were cultured with dilutions of products or placebo for 24 hours. After washing, culture viability was determined using Cell Titer$\mathrm{Glo}^{\mathrm{TM}}$ assay to the manufacturere's instructions. The dilutions of the products or placebo that resulted in $\geq 60 \%$ culture viability were presented.

Table 1. Nontoxic dilutions of the candidate microbicide and placebo formulations in epithelial cell lines and primary immune cells1.

\begin{tabular}{llll}
\hline Products & $\begin{array}{l}\text { Epithelial Cell } \\
\text { Lines-Caco2 }\end{array}$ & $\begin{array}{l}\text { Primary immune } \\
\text { Cells-PMBCs }\end{array}$ & $\begin{array}{l}\text { Primary Immune } \\
\text { Cells-MФ }\end{array}$ \\
\hline KY-N9 & $1: 1000$ & $1: 1000$ & $1: 1000$ \\
KY jelly & $1: 100$ & $1: 100$ & $1: 100$ \\
\hline
\end{tabular}

\section{Results and discussion}

The KY and N9 formulations were much more toxic, and when tested at their nontoxic concentrations against primary immune cells PBMCs and Macrophages and epithelial cells. Originally developed as a spermicide, initial reports showed N9 had in vitro activity against sexually transmitted diseases including HIV-1 [21,22]. Despite this promising early work, one study reported that the $\mathrm{N} 9$ antiviral activity only occurred at doses that were cytotoxic [23]. Several clinical studies have reported epithelial disruption and inflammation of the female genital tract $[24,25]$ and two additional studies showed toxicity of the rectal mucosa in humans and nonhuman primates $[26,27]$. Since that time, the recommendation has been that products containing N9 not be used for HIV-1 prevention, especially rectally. Our data were consistent with these results showing that KY-N9 was highly toxic to the cells tested and had 
reduced efficacy in blocking HIV-1 infection at its nontoxic concentration.

Inflammation of the female reproductive tract increases susceptibility to HIV and other viral infections and, thus, it becomes a serious liability for vaginal products. Excessive release of proinflammatory cytokines may alter the mucosal balance between tissue destruction and repair and linked to enhanced penetration and replication of viral pathogens upon chemical insult.

We have placed the emphasis on value of interleukin (IL-)$1 \beta$, TNF- $\alpha$, IL- 6 and IL- 8 as marker of mucosal toxicity because they represent three families of cytokines with different roles in inflammatory tissue damage and HIV-1 pathogenesis. IL-1 $\beta$, TNF- $\alpha$, and IL- 6 induce HIV-1 expression via NF- $\kappa$ B mediated HIV-1 long terminal repeat (LTR) activation, while IL-8 triggers the recruitment of HIV-1 susceptible cells to the inflammation site and may also stimulate HIV-1 replication in T cells and macrophages [28]. All of these three cytokines are expressed constitutively at low levels by cervico vaginal keratinocytes and are significantly induced via NF- $\mathrm{KB}$ mediated signal transduction pathways by proinflammatory stimuli and common sexually transmitted pathogens [28].

In this study, we have investigated the correlation between the response of peripheral blood mononuclear cells (PBMCs) and macrophages from non-infected cells (Figure 1-4). As a result, PBMCs and macrophages were exposed to non-toxic according to microbicides dilutions. Higher concentrations proinflammatory cytokines in genital secretions have been associated with higher levels of vaginal virus in HIV-1 infected women. Therefore, it will be important to know if non-toxic levels of the microbicides increase production of these cytokines [29].

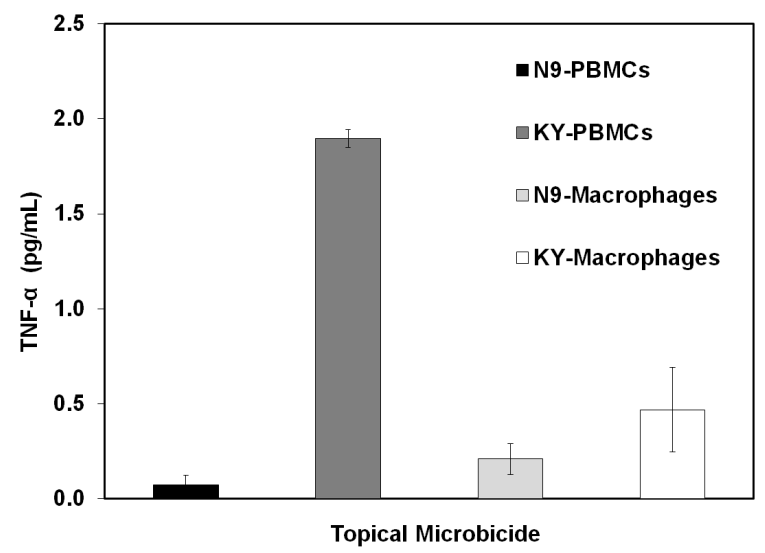

Figure 1. The ability of topical microbicide formulation to induce proinflammatory cytokine TNF- $\alpha$ in peripheral mononuclear cells (PBMCS) and macrophages. PBMCS and macrophages were suspended in $1 \mathrm{~mL}$ media mixed with non-toxic formulations of microbicides for $4 \mathrm{hrs}$. The products were tested in triplicate. Post exposure to microbicides, the supernatant were collected and assayed for cytokine-TNF- $\alpha$ release using ELISA (R \& D systems). The data presented are average \pm standard deviation of the means.

Currently, there is no commercially available microbicide with anti-HIV activity. However, it is a pivotal time in HIV microbicide research, in which more resources are becoming available and progress is being made in the field. For example, the well-known spermicidal Nonoxynol-9 (N9) had been shown to inactivate HIV-1 [2] and thus became popular for use in safesex practices. Recent studies, however, have shown that N-9 actually increases the risk of HIV-1 transmission [3], due to increased HIV-1 shedding and increase recruitment of $\mathrm{CD}+4$ target cells associated with vaginal inflammation. In addition, $\mathrm{N}-9$ use promotes HIV-1 transmission through interleukinmediated NF- $\mathrm{\kappa B}$ activation, and increased replication [30]. In this study, N-9 showed levels of TNF- $\alpha$, IL-1 $\beta$ and IL-6 cytokines were $<2 \mathrm{pg} / \mathrm{mL}$, whereas elevated level of IL-8 has been observed in our in vitro study. In one study, showed that $\mathrm{N}-9$ induced IL- $\alpha / \beta$ release, activation if NF- $\kappa B$ and subsequent IL-8 up-regulation by cervical and vaginal epithelial in vitro [32]. Also in one study shows the effects of formulations containing the common spermicide active ingredient, nonoxynol-9 (N9), on epithelial tissue containing epithelial VEC cells, with increasing $\mathrm{N} 9$ concentration, the tissue viability decreases and the IL-1 $\beta$ release increase. Such assays can be used to predict toxicity of vaginal care products, microbicides and other chemical agents.

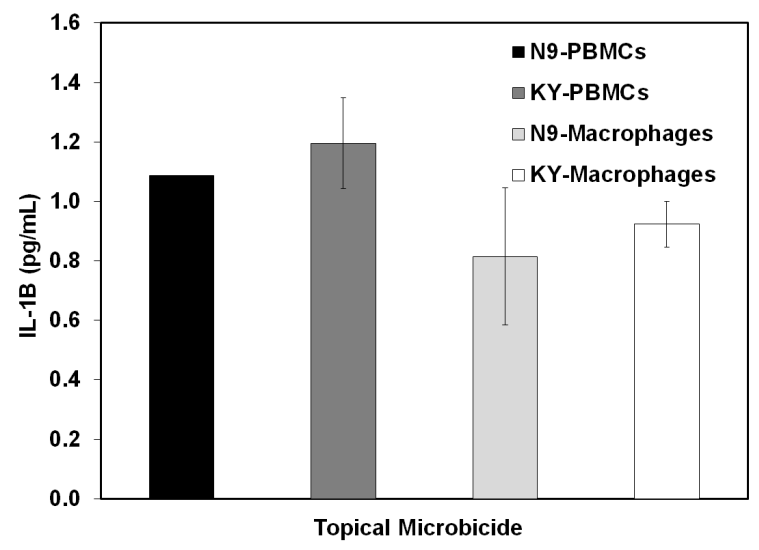

Figure 2. The ability of topical microbicide formulation to induce proinflammatory cytokine IL- $1 \beta$ in peripheral mononuclear cells (PBMCS) and macrophages. PBMCS and macrophages were suspended in $1 \mathrm{~mL}$ media mixed with non-toxic formulations of microbicides for $4 \mathrm{hrs}$. The products were tested in triplicate. Post exposure to microbicides, the supernatant were collected and assayed for cytokine IL-1 $\beta$ release using ELISA (R \& D systems). The data presented are average \pm standard deviation of the means.

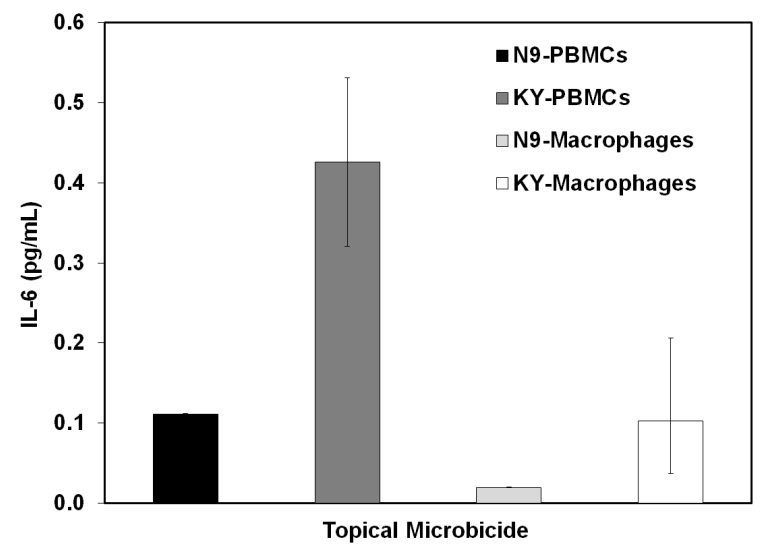

Figure 3. The ability of topical microbicide formulation to induce proinflammatory cytokine IL-8 in peripheral mononuclear cells (PBMCS) and macrophages. PBMCS and macrophages were suspended in $1 \mathrm{~mL}$ media mixed with non-toxic formulations of microbicides for $4 \mathrm{hrs}$. The products were tested in triplicate. Post exposure to microbicides, the supernatant were collected and assayed for cytokine IL-8 release using ELISA (R \& D systems). The data presented are average \pm standard deviation of the means.

\section{Conclusion}

In conclusion all microbicides tested in their non-toxic formulation in the two models of PBMCs and macrophages showed relatively low levels of IL-1b, TNF- $\alpha$ and IL-6. This indicated safety and low toxicity of these microbicide in terms of cytokines release. Also, the usage of these microbicieds as anti-HIV formulation will not trigger cytokine release. On the other hand, IL has shown relatively higher levels in all microbicide tested in their non-toxic formulation. This 
indicated that microbicide induced the release of IL-8 could trigger the recruitment of HIV-1 susceptible cells and increase HIV-1 replication. The only way to support these results by clinical trials including phase-1 safety studies.

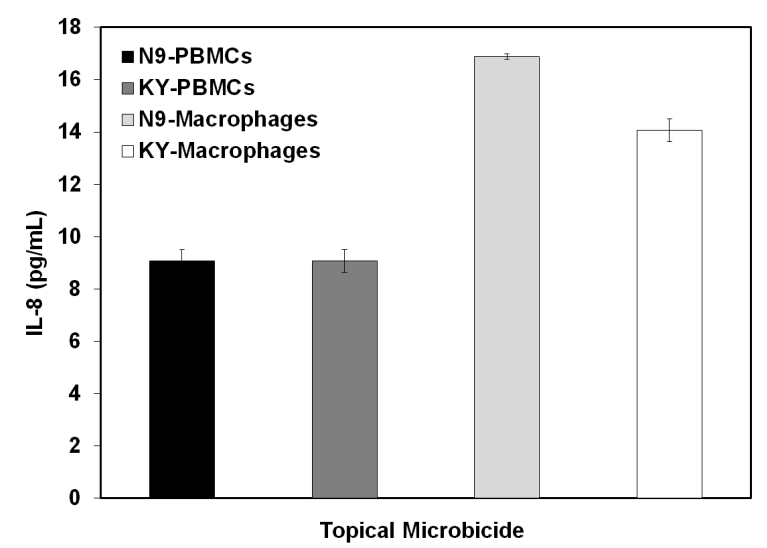

Figure 4. The ability of topical microbicide formulation to induce proinflammatory cytokine IL-6 in peripheral mononuclear cells (PBMCS) and macrophages. PBMCS and macrophages were suspended in $1 \mathrm{~mL}$ media mixed with non-toxic formulations of microbicides for $4 \mathrm{hrs}$. The products were tested in triplicate. Post exposure to microbicides, the supernatant were collected and assayed for cytokineIL- 6 release using ELISA (R \& D systems). The data presented are average \pm standard deviation of the means.

\section{Acknowledgements}

We thank Dr. Martin D'Souza for his technical assistance and all other individual in the center for drug deliver at Mercer University.

\section{References}

[1]. Who Notes and News, HIV/AIDS, World Health Forum 1997, 18(3-4), 369-372.

[2]. Bird, K. D. AIDS 1991, 5, 791-796

[3]. Stephenson, J. JAMA 2000, 284, 949-949.

[4]. Zacharapoulos, V.; Phillips, D. Clin. Diagn. Lab. Immunol. 1997, 4(4), 465-468.

[5]. Baron, S.; Poast, J.; Nguyen, D.; Cloyd, M. W. AIDS Res. Hum. Retroviruses 2001, 17, 997-1002.

[6]. Milman, G.; Sharma, O. AIDS Res. Hum. Retroviruses 1994, 10, 13051312.

[7]. Bomsel, M. Nat. Med. 1997, 3, 42-47.

[8]. Zacharopoulos, V. R.; Phillips, D. M. Microb. Pathog. 1997, 23, 225-233.

[9]. Phillips, D. M.; Bourinbaiar, A. S. Virology 1992, 186, 261-273.

[10]. Tan, X.; Phillips, D. M. Arch. Virol. 1996, 141, 1177-1189.

[11]. Smith, P. D.; Li, L.; Meng, G. J. Infect. Dis. 1999, 179, S436-S440.

[12]. Kabat, D.; Kozak, S. L.; Wehrly, K.; Chesebro, B. J. Virol. 1994, 68, $2570-$ 2577.

[13]. Ariyoshi, K.; Harwood, E.; Chiengsong-Popov, R.; Weber, J. Lancet 1992, 340, 1257-1258.

[14]. Clark, S. J.; Saag, M. S.; Decker, W. D. Campbell-Hill, S.; Roberson, J. L.; Veldkamp, P. J.; Kappes, J. C.; Hahn, B. H.; Shaw, G. M. N. Engl. J. Med. 1991, 324, 954-960.

[15]. Daar, E. S.; Moudgil, T.; Meyer, R. D.; Ho, D. D. N. Engl. J. Med. 1991, 324, 961-964.

[16]. Connick, E.; Marr, D. G.; Zhang, X. Q.; Clark, S. J.; Saag, M. S.; Schooley, R. T.; Curiel, T. J. AIDS Res. Hum. Retroviruses 1996, 12, 1129-1140.

[17]. Lawn, S. D.; Subbarao, S.; Wright, Jr. T. C.; Evans-Strickfaden, T.; Ellerbrock, T. V.; Lennox, J. L.; Butera, S. T.; Hart, C. E. J. Infec. Diseases 2000, 181, 1950-1956.

[18]. Sykes, J. A.; Whitescarver, J.; Jernstrom, P.; Nolan, J. F.; Bayatt, P. J. Natl. Cancer Inst. 1970, 45, 107-122.

[19]. Huramato, H. Acta Obstet. Gynaecol. Jpn. 1972, 19, 47-58.

[20]. Fogh, J.; Wright, W. C.; Loveless, J. D. J. Natl. Cancer Inst. 1977, 58, $209-$ 214.

[21]. Jennings, R.; Clegg, A. J. Antimicrob. Chemother. 1993, 32, 71-82.

[22]. Malkovsky, M.; Newell, A.; Dalgleish, A. G. Lancet 1988, 1, 645-645.

[23]. Patton, D. L.; Kidder, G. G.; Sweeney, Y. C.; Rabe, L. K.; Clark, A. M.; Hillier, S. L. Sex. Transm. Dis. 1996, 23, 461-464.

[24]. Stafford, M. K.; Ward, H.; Flanagan, A.; Rosenstein, I. J.; TaylorRobinson, D.; Smith, J. R.; Weber, J.; Kitchen, V. S. J. Acquir. Immune Defic. Syndr. Hum. Retrovirol. 1998, 17, 327-331.
[25]. Van Damme, L.; Ramjee, G.; Alary, M.; Vuylsteke, B.; Chandeying, V.; Rees, H.; Sirivongrangson, P.; Mukenge-Tshibaka, L.; Ettiegne-Traore, V.; Uaheowitchai, C.; Karim, S. S.; Masse, B.; Perriens, J.; Laga, M. Lancet 2002, 360, 971-977.

[26]. Patton, D. L.; Cosgrove Sweeney, Y. T.; Rabe, L. K.; Hillier, S. L. Sex. Transm. Dis. 2002, 29, 581-587.

[27]. Phillips, D. M.; Taylor, C. L.; Zacharopoulos, V. R.; Maguire, R. A. Contraception 2000, 62, 149-154.

[28]. Fichorova, R. N.; Zhou, F.; Ratnam, V.; Atanssova, V.; Jiang, S.; Strick, N.; Neurath, A. R. Antimicrob. Agents Ch. 2005, 323-335.

[29]. Centers for Disease Control and Prevention (CDC). National Center for HIV, STD and TB prevention. August 2000.

[30]. Fichorova, R. N.; Tucker, L. D.; Anderson, D. J. J. Inect. Dis. 2001 184(4), 418-428. 